\title{
Countable families of solutions of a limit stationary semilinear fourth-order Cahn-Hilliard-type equation I. Mountain pass and Lusternik-Schnirel'man patterns in $\mathbb{R}^{N}$
}

\author{
P Álvarez-Caudevilla ${ }^{1,2^{*}}$ (D, JD Evans ${ }^{3}$ and VA Galaktionov ${ }^{3}$
}

\begin{tabular}{l}
\hline "Correspondence: \\
pacaudev@math.uc3m.es \\
1Universidad Carlos III de Madrid, \\
Av. Universidad 30, Leganés, 28911, \\
Spain \\
2Instituto de Ciencias Matemáticas, \\
ICMAT (CSIC-UAM-UC3M-UCM), \\
C/Nicolás Cabrera 15, Madrid, \\
28049, Spain \\
Full list of author information is \\
available at the end of the article
\end{tabular}

available at the end of the article

\begin{abstract}
Solutions of the stationary semilinear Cahn-Hilliard-type equation

$$
-\Delta^{2} u-u-\Delta\left(|u|^{p-1} u\right)=0 \text { in } \mathbb{R}^{N} \text {, with } p>1
$$

which are exponentially decaying at infinity, are studied. Using the mounting pass lemma allows us to determinate the existence of a radially symmetric solution. On the other hand, the application of Lusternik-Schnirel'man (L-S) category theory shows the existence of, at least, a countable family of solutions.

However, through numerical methods it is shown that the whole set of solutions, even in 1D, is much wider. This suggests that, actually, there exists, at least, a countable set of countable families of solutions, in which only the first one can be obtained by the L-S min-max approach.
\end{abstract}

MSC: $35 \mathrm{G} 20 ; 35 \mathrm{~K} 52$

Keywords: stationary Cahn-Hilliard equation; variational setting; non-unique oscillatory solutions; countable family of critical points

\section{Introduction and motivation for main problems}

\subsection{Models and preliminaries}

This paper studies some multiplicity properties of the steady-states of the following fourthorder parabolic equation of the Cahn-Hilliard (C-H) type:

$$
u_{t}=-\Delta^{2} u-u-\Delta\left(|u|^{p-1} u\right) \quad \text { in } \mathbb{R}^{N} \times \mathbb{R}_{+} \text {, where } p>1 \text {. }
$$

The problem is completed with bounded smooth initial data,

$$
u(x, 0)=u_{0}(x) \text { in } \mathbb{R}^{N}
$$

Assuming that data $u_{0}(x)$ are sufficiently fast exponentially decaying at infinity, the same behaviour holds for the unique classic solution of (1.1), at least locally in time, since, for $p>1, u(x, t)$ may blow-up in finite time; see key references and results in [1] and [2].

(c) 2016 Álvarez-Caudevilla et al. This article is distributed under the terms of the Creative Commons Attribution 4.0 International License (http://creativecommons.org/licenses/by/4.0/), which permits unrestricted use, distribution, and reproduction in any medium, provided you give appropriate credit to the original author(s) and the source, provide a link to the Creative Commons license, and indicate if changes were made. 
The classic Cahn-Hilliard equation describes the dynamics of pattern formation in phase transition in alloys, glasses, and polymer solutions. When a binary solution is cooled sufficiently, phase separation may occur and then proceed in two ways: either nucleation, in which nuclei of the second phase appear randomly and grow, or, in the so-called spinodal decomposition, the whole solution appears to nucleate at once and then periodic or semi-periodic structures appear. Pattern formation resulting from a phase transition has been observed in alloys, glasses and polymer solutions. Mathematically the classic CahnHilliard equation reads

$$
u_{t}-\Delta K(u)=0 \quad \text { in } \mathbb{R}^{N} \times \mathbb{R}_{+} \text {, where } K(u):=-v \Delta u+f(u), v>0,
$$

with the unknown function $u=u(x, t)$ is a scalar for any $x \in \mathbb{R}^{N}, t \in \mathbb{R}_{+}$. Then, in general, the function $f(u)$ is a polynomial of the order $2 p-1$,

$$
f(u):=\sum_{j=1}^{2 p-1} a_{j} u^{j}, \quad p \in \mathbb{N}, p \geq 2 .
$$

In particular, the classic Cahn-Hilliard equation corresponds to the case $p=2$ and

$$
f(u):=-\eta u+\mu u^{3}, \quad \eta, \mu>0 .
$$

Moreover, Cahn-Hilliard equations-type such as (1.1) have been studied by many authors during the last decades, especially for non-negative solutions. Among some of the works where several aspects related to these equations are [3-7]. Additionally, one can check the surveys in $[2,8]$, where necessary aspects of global existence and blow-up of solutions for (1.1) are discussed in sufficient detail.

Paralelly, these types of equations possess a great interest in biology and, after certain transformations (see below) as prototype for nerve conduction in the form of a FitzHughNagumo system which is represented by a non-cooperative system $(c f .[9,10])$. This is actually a great motivation for equation (1.1) presented here because the direct connection between equation (1.1) and a class of non-cooperative systems; see details and further discussion below.

From the mathematical point of view, the non-stationary equation (1.1) involves a fourth-order elliptic operator and it contains a negative viscosity term and that is the reason because we say that (1.1) is a Cahn-Hilliard equations-type. Hence, although both equations have a non-linearity depending on a second-order operator here we consider solutions of changing sign.

Many questions still remain unanswered for equations of this type, as well as noncooperative systems, especially in relation to multiplicity problems (see details below and references). Here we show some existence and multiplicity results for equation (1.1), which might be applied for other models such as the classical Cahn-Hilliard equation (1.3). Additionally we have found numerically some surprising chaotic oscillatory patterns.

\subsection{Variational approach and main results}

Thus, we concentrate on the analysis of multiplicity results for the fourth-order elliptic stationary Cahn-Hilliard equation

$$
-\Delta^{2} u-u-\Delta\left(|u|^{p-1} u\right)=0 \quad \text { in } \mathbb{R}^{N} \text {, where } p>1 .
$$


Note that (1.4) is not variational in $L^{2}\left(\mathbb{R}^{N}\right)$, though it is variational in $H^{-1}\left(\mathbb{R}^{N}\right)$. Hence, multiplying $(1.4)$ by $(-\Delta)^{-1}$, we obtain an elliptic equation with a non-local operator of the form

$$
-\Delta u+(-\Delta)^{-1} u-|u|^{p-1} u=0
$$

Here, as customary, $(-\Delta)^{-1} u=v$, if

$$
-\Delta v=u \quad \text { in } \mathbb{R}^{N}, \quad v(x) \rightarrow 0 \quad \text { as } x \rightarrow \infty .
$$

This yields the following $C^{1}$-functional associated with (1.5):

$$
\mathcal{F}(u):=\frac{1}{2} \int_{\mathbb{R}^{N}}|\nabla u|^{2}+\frac{1}{2} \int_{\mathbb{R}^{N}}\left|(-\Delta)^{-\frac{1}{2}} u\right|^{2}-\frac{1}{p+1} \int_{\mathbb{R}^{N}}|u|^{p+1} .
$$

Solutions of equation (1.5) are then obtained as critical points of the functional (1.6), assuming them to be radially symmetric and belonging to the space where

$$
\int_{\mathbb{R}^{N}}\left|(-\Delta)^{-\frac{1}{2}} u\right|^{2}<\infty
$$

In other words, we are looking for solutions belonging to the space $\mathbf{H}$ denoted by

$$
\mathbf{H}=\left\{u \in W_{\mathrm{rad}}^{1,2}\left(\mathbb{R}^{N}\right): \int_{\mathbb{R}^{N}}\left|(-\Delta)^{-\frac{1}{2}} u\right|^{2}<\infty\right\}
$$

where $W_{\mathrm{rad}}^{1,2}\left(\mathbb{R}^{N}\right)$ is the classical Sobolev space constrained to radially symmetric functions. The non-local operator $(-\Delta)^{-1}$ is a positive linear integral operator from $L^{q}\left(\mathbb{R}^{N}\right)$ to $L^{2}\left(\mathbb{R}^{N}\right)$, with $q=\frac{2 N}{N+4}$. Moreover, the operator $(-\Delta)^{-\frac{1}{2}}$ can be correctly defined as the square root of the operator $(-\Delta)^{-1}$ and it will also be referred to as a non-local linear operator.

Also, since the problem is set in $\mathbb{R}^{N}$ we are defining this operator in a class of exponentially decaying functions; see below the details and conditions to have the weak expression of the problem with these exponentially decay solutions.

A similar fourth-order problem was studied in [1], where further references can be found. However, in [1] the stationary equation (1.4) was considered in a bounded smooth domain $\Omega \subset \mathbb{R}^{N}$, with homogeneous Navier-type boundary conditions

$$
u=\Delta u=0 \quad \text { on } \partial \Omega .
$$

In particular, it was shown that this problem (1.4), (1.8) admits a countable family of solutions (critical points/values). Moreover, in Section 6 of [11] for a different variational fourth-order problem in $\mathbb{R}$, it was shown that a wider countable set of countable families of solutions can be expected, where only the first infinite family is the Lusternik-Schnirel'man one.

Hence, due to the analysis performed in $[11,12]$ we need to study, in a general multidimensional geometry, existence and multiplicity for the elliptic equation (1.4) in a class of functions properly decaying at infinity (in fact exponentially),

$$
\lim _{|x| \rightarrow \infty} u(x)=0
$$

In particular, radial solutions that belong to the space $\mathbf{H}$ denoted by (1.7). 
Subsequently, dealing with radial solutions in $\mathbb{R}^{N}$ the Sobolev space $W_{\text {rad }}^{1,2}\left(\mathbb{R}^{N}\right)$ is compactly imbedded into $L^{p+1}\left(\mathbb{R}^{N}\right)$ in the subcritical Sobolev's range

$$
1<p \leq p_{*}=\frac{N+2}{N-2}\left(\equiv \frac{2 N}{N-2}-1=p_{\mathrm{S}}\right)-1, \quad N \geq 3\left(p_{*}=+\infty \text { for } N=1,2\right) .
$$

In other words,

$$
W_{\mathrm{rad}}^{1,2}\left(\mathbb{R}^{N}\right)=H_{\mathrm{rad}}^{1}\left(\mathbb{R}^{N}\right) \hookrightarrow L^{p+1}\left(\mathbb{R}^{N}\right) \Leftrightarrow 1<p<p_{*} .
$$

See [13] for further details. Note that, here, for the fourth-order elliptic operator in (1.4), $p_{\mathrm{S}}=\frac{2 N}{N-2}$, with $N \geq 3$, because this operator has the representation

$$
-\Delta^{2} u-\Delta\left(|u|^{p-1} u\right)=-\Delta\left(\Delta u+|u|^{p-1} u\right),
$$

so that the necessary embedding features are governed by a standard second-order one

$$
\Delta u+|u|^{p-1} u
$$

Since the category of the functional subset increases without bound in such a limit, eventually, we then expect to arrive at, at least, a countable family of various critical values/points.

However, here we perform a variational study directly in $\mathbb{R}^{N}$, which was done previously for many fourth-order ODEs and elliptic equations; see [14-16] as key examples (though those equations, mainly, contain coercive operators, with 'non-oscillatory' behaviour at infinity).

Namely, by a linearised analysis we first check that equation (1.4) provides us with a sufficient 'amount' of exponentially decaying solutions at infinity. Obviously, in any bounded class of such functions, and in a natural functional viewing, since, loosely speaking, nothing happens at infinity (effectively, the solutions vanish there), the variational problem can be treated as the one in a bounded domain. So that the embedding in (1.11) comes in charge in some sense.

Remark 1.1 As mentioned above, throughout this paper we shall consider radial solutions. Hence, we take into consideration a well-known result about continuous Sobolev's embedding (see for instance [17]). To be precise, we will consider the embedding

$$
W_{\mathrm{rad}}^{2,2}\left(\mathbb{R}^{N}\right) \hookrightarrow L^{p+1}\left(\mathbb{R}^{N}\right),
$$

which is continuous for every $1 \leq p \leq p_{*}$ where

$$
p_{*}=\frac{N+4}{N-4}=\frac{2 N}{N-4}-1=p_{\mathrm{S}}-1, \quad N \geq 5 \quad \text { and } \quad p_{*}=+\infty \quad \text { for } N=1,2,3,4 .
$$

On the other hand, this embedding will be compact if in addition $2 \leq N$ and $p<p^{*}$ (see [18]). Thus, there is a constant $S_{N}>0$ such that

$$
\|u\|_{L^{p+1}\left(\mathbb{R}^{N}\right)} \leq S_{N}\|u\|_{W_{\mathrm{rad}}^{2,2}\left(\mathbb{R}^{N}\right)}
$$


Let us briefly summarise what we obtain here. First, we perform an analysis based on the application of the mountain pass theorem in order to ascertain the existence of one radial solution and, eventually, through a Lusternik-Schnirel'man argument the existence of more than one for equation (1.4).

Thus, we state (proved in Section 3) the following.

Theorem 1.1 Suppose $N \geq 2$. Then the non-local equation (1.5) possesses at least one solution in the space $\mathbf{H}$, denoted by (1.7), with exponential decay and for exponents $p$ 's in the Sobolev range (1.10).

As far as we know, to get the existence of more than one solution, this seems to be the best available approach, since, for this type of higher-order PDEs, we have a big lack of classical methodology and PDE theory.

In addition, secondly, we apply a L-S-fibering approach to get a countable family of solutions (critical points), though without any detailed information as regards how they look like. Indeed, we cannot be assured if the solutions obtained using the L-S theory are radially symmetric or not.

Therefore we state and prove in Section 4 the following.

Theorem 1.2 Suppose $N \geq 2$. Then there is a countable family of solutions for the nonlocal equation (1.5) of the L-S type.

Finally, we apply advanced numerical methods to describe general 'geometric' structure of various solutions assuming symmetric for even profiles and anti-symmetric conditions for odd profiles (see below). In particular, we introduce some chaotic patterns for equation (1.4) for $p=3$ and $p=2$, showing some profiles that become very chaotic away from the point of symmetry.

These numerical experiments suggest that the whole set of solutions, even in 1D, is much wider. However, these numerical experiments, together with some analytical approaches and estimates, will be extended and analysed in more detail in [19]. Indeed, it will be proved there that there exists, at least, a countable set of countable families of critical points in which, only the first one can be obtained by the L-S min-max approach. Note that we have used the numerical analysis in 1D just to show the existence of other families of solutions. Something that has not been proved here analytically.

Also, we observe that performing numerical experiments, shooting smoothly from $x=$ 0 with $u^{\prime}(0)=u^{\prime \prime}(0)=u^{\prime \prime \prime}(0)=0$ and varying $u(0)$, those chaotic patterns become more periodic when $u(0)$ increases. Indeed, this fact appears to be sooner for $p=3$ than for $p=2$.

Moreover, it should be mentioned that this kind of transition behaviour is seen in similar phase solidification fourth-order equations, such as the Kuromoto-Sivashinsky and SwiftHohenberg equations [20,21], as the critical order parameter increases.

\subsection{Previous related results}

Recall again that, in [1], existence and multiplicity results were obtained for the steadystates of the unstable $\mathrm{C}-\mathrm{H}$ equation of the form

$$
-\Delta^{2} u+\gamma u-\Delta\left(|u|^{p-1} u\right)=0 \quad \text { in } \Omega(p>1)
$$


for a real parameter $\gamma$, where the multiplicity essentially depended on this parameter, which affected the category of the functional subset associated with the principal linear operator. The analysis was based on variational methods such as the fibering method, potential operator theory and Lusternik-Schnirel'man category-genus theory, and others, such as homotopy approaches or perturbation theory existence. Specifically, it was found that, depending on the parameter $\gamma$, there exist a different number of stationary solutions, i.e.:

- If the parameter $\gamma \leq K \lambda_{1}$, with $K>0$ a positive constant and $\lambda_{1}>0$ the first eigenvalue of the bi-harmonic operator, i.e., $\Delta^{2} \varphi_{1}=\lambda_{1} \varphi_{1}$ with Navier boundary conditions (1.8), then there exists at least one solution for the equation (1.14).

- When the parameter is greater than the first eigenvalue of the bi-harmonic equation $\lambda_{1}$, multiplied by the positive constant $K$, then there will not be any solution at all, if one assumes only positive solutions. However, for oscillatory solutions of changing sign the number of possible solutions increases with the value of the parameter $\gamma$. In fact, when the parameter $\gamma$ goes to infinity, one has an arbitrarily large number of distinct solutions.

Remark 1.2 Note that the previous distinction in terms of the number of solutions is related to pattern formation of problems such as the Kuramoto-Sivashinsky equation; see $[20,22]$ for further references and details.

Furthermore, the so-called mountain pass theorem [23] has been previously applied to get some multiplicity results. Indeed, in [24] the authors analysed the existence of a second solution for the fourth-order elliptic problem

$$
\left\{\begin{array}{l}
-\Delta^{2} u-c_{1} \Delta u+c_{2} u=u^{p}+k \sum_{i=1}^{m} \alpha_{i} \delta_{a_{i}} \quad \text { in } \mathcal{D}^{\prime}\left(\mathbb{R}^{N}\right), \\
u(x)>0, \quad u(x) \rightarrow 0 \quad \text { as }|x| \rightarrow 0,
\end{array}\right.
$$

where $\delta_{a_{i}}$ represents the Dirac delta function supported at $a_{i} \in \mathbb{R}^{N}$ and

$$
1<p<\frac{N}{N-4}, \quad c_{1}^{2}-4 c_{2} \geq 0,
$$

and with $N \geq 5, k>0, m \in \mathbb{N}, \alpha_{i}>0, c_{1}, c_{2}>0$.

Other methods utilised to solve similar problems to (1.4), such as

$$
\Delta^{2} u+\beta^{2} \Delta u+u=f(u)
$$

might be Hamiltonian methods or the strong maximum principle. The previous equation can be written by

$$
\left(\Delta-\mu_{1}\right)\left(\Delta-\mu_{2}\right) u=f(u)
$$

where $\mu_{1}$ and $\mu_{2}$ are the roots of the characteristic polynomial

$$
\mu^{2}+\beta^{2} \mu+1=0, \quad \text { given by } \quad \mu_{i}=\frac{1}{2}\left(-\beta^{2} \pm \sqrt{\beta^{4}-4}\right), \quad i=1,2 .
$$


Then, if $\beta \geq \sqrt{2}$ both roots are real and positive we can write equation (1.16) as the system

$$
\left\{\begin{array}{l}
\Delta u-\mu_{1} u=v \\
\Delta v-\mu_{2} v=f(u)
\end{array}\right.
$$

and apply the strong maximum principle having a positive solution

$$
u(x)>0 \text { for } x \in \mathbb{R}^{N},
$$

if we assume that $u$ is a homoclinic orbit (see $[16,25]$ for any further details with $N=1$ ). Moreover, in this case one can ascertain certain qualitative properties for that homoclinic orbit, such as the existence of a precisely critical point for $u, a$ priori estimates and the symmetry of $u$ with respect to that critical point. However, if $0<\beta<\sqrt{2}$, one cannot obtain such a decoupling.

Also, similar fourth-order equations to $(1.16)$ have been analysed in $[14,15]$ in the dimensional space $\mathbb{R}^{4}$ and via Hamiltonian methods obtaining the connection between the critical points of the Hamiltonian.

On the other hand, note that problem (1.4) can be written as the following elliptic system:

$$
\left\{\begin{array}{l}
-\Delta u=-v-|u|^{p-1} u, \quad \text { in } \mathbb{R}^{N}, \\
-\Delta v=u
\end{array}\right.
$$

where $v:=(-\Delta)^{-1} u$, which gives a different perspective to the problem in hand. The solutions of the system (1.18) represent the steady-states of a reaction-diffusion system of the form

$$
\left\{\begin{array}{l}
u_{t}-\Delta u=-v-|u|^{p-1} u, \\
v_{t}-\Delta v=u
\end{array} \quad \text { in } \mathbb{R}^{N},\right.
$$

with a great interest in biology and as prototype for nerve conduction in the form of a FitzHugh-Nagumo system $(c f .[9,10])$. Mathematically, we note that system $(1.18)$ is weakly coupled. However, this coupling is non-cooperative, in other words, the coupled terms have different signs. This means that the maximum principle is not valid for the system (1.18). For a cooperative system where the maximum principle can be applied to a very similar system to (1.18) see [26].

Basically, so far, one can see that, due to the lack of comparison methods, maximum principle, and several others classical methods in the analysis of higher-order PDEs different approaches must be followed in order to get new results for this type of higher-order PDEs. Therefore, in general the methodology is very limited and restricted to very specific examples.

\subsection{Further extensions}

Our results can be applied to other $\mathrm{C}-\mathrm{H}$ models. For example note that, for the 'true' fourth-order semilinear operator, the critical Sobolev's exponent is different:

$$
-\Delta^{2} u+|u|^{p-1} u=0 \quad \Longrightarrow \quad p_{\mathrm{S}}=\frac{N+4}{N-4} \quad \text { for } N \geq 5 .
$$


Obviously, a critical Sobolev's range as in (1.20) occurs for a different sixth-order C-H equation

$$
\Delta^{3} u \pm u-\Delta\left(|u|^{p-1} u\right)=0 \quad \text { in } \mathbb{R}^{N}
$$

However, if the unstable nonlinear diffusion operator is of fourth order, as in

$$
\Delta^{3} u \pm u+\Delta^{2}\left(|u|^{p-1} u\right)=0 \quad \text { in } \mathbb{R}^{N}
$$

we again arrive at the 'second-order' Sobolev range as in (1.10).

Equations (1.21) and (1.22) can be studied along similar lines, but some aspects become more technical, though not affecting the principal conclusions and results.

\section{Preliminary results: exponentially decaying patterns in $\mathbb{R}^{N}$}

\subsection{Exponentially decaying patterns in $\mathbb{R}^{N}$}

The preliminary conclusions presented here formally allow us to consider our equations (1.4) in the whole $\mathbb{R}^{N}$, unlike as in [1] where the problem was assumed to be in a bounded domain $\Omega \subset \mathbb{R}^{N}$.

Indeed, for the functional (1.6) we deal with the integrals over $\mathbb{R}^{N}$ and, actually, with the functional setting over a certain weighted Sobolev space, ${ }^{\text {a }}$ instead of $W_{0}^{2,2}(\Omega)$ as assumed in [1]. Such a functional setting of the problem in $\mathbb{R}^{N}$ is key in the following. In fact, a proper functional setting assumes certain admissible asymptotic decay of solutions at infinity, which, for (1.4), is governed by the corresponding linearised operator.

Thus, considering (1.4) in the radial geometry, with $u=u(r)$ and $r=|x| \geq 0$, we then obtain

$$
\begin{aligned}
\Delta^{2} u & \equiv u^{(4)}+\frac{2(N-1)}{r} u^{\prime \prime \prime}+\frac{(N-1)(N-3)}{r^{2}} u^{\prime \prime}-\frac{(N-1)(N-3)}{r^{3}} u^{\prime} \\
& =-u-p|u|^{p-3} u\left(u u^{\prime \prime}+(p-1)\left(u^{\prime}\right)^{2}+\frac{(N-1)}{r} u u^{\prime}\right) .
\end{aligned}
$$

Next, as usual, calculating the admissible decaying asymptotics from (2.1), using a two scale WKBJ-type asymptotics, as a first approximation (sufficient for our purposes), we use an exponentially pattern of the form $u(r) \approx r^{\delta} \mathrm{e}^{a r}$ (as $r \rightarrow \infty$ ) in (2.1) leading easily to the following characteristic equation:

$$
a^{4}=-1 \quad \text { and } \quad \delta=-\frac{N-1}{2} \text {. }
$$

To be precise, note that the first equation in (2.2) comes from the homogeneity of the leading terms in (2.1). The second equality in (2.2) comes from a similar argument after evaluating the next leading terms on the left-hand side in (2.1). This yields a two-dimensional exponential bundle:

$$
u(r) \approx r^{-\frac{N-1}{2}} \mathrm{e}^{-r / \sqrt{2}}\left[C_{1} \cos \left(\frac{r}{\sqrt{2}}\right)+C_{2} \sin \left(\frac{r}{\sqrt{2}}\right)\right]
$$

where $C_{1,2} \in \mathbb{R}$ are two arbitrary parameters of this linearised bundle. 
Through this asymptotic analysis we shall be able to show some patterns after performing a shooting problem in Section 5.

Let us now perform a preliminary analysis. In this radial geometry, any regular bounded solution of (1.4) must satisfy two boundary conditions at the origin (making the biLaplacian non-singular)

$$
u^{\prime}(0)=u^{\prime \prime \prime}(0)=0
$$

Hence, using a standard shooting strategy from $r=+\infty$, algebraically, at least two parameters are needed to satisfy both (2.4).

Looking again at (2.3), where there exist $t$ wo parameters $C_{1,2} \in \mathbb{R}$, we observe that matching with two symmetry boundary conditions (2.4) yields a well-posed and well-balanced algebraic '2D-2D shooting problem'.

To justify existence of such solutions (critical points), we then need carefully apply different variational techniques. Of course, for the elliptic problem in $\mathbb{R}^{N}$, one needs a more technical and delicate 'asymptotic separation' analysis. Namely, one needs to resolve the following 'separation' procedure (as is well known, for the bi-Laplacian, a standard separation of variables is not available):

$$
\Delta^{2} u \equiv\left(\Delta_{r}+\frac{1}{r^{2}} \Delta_{\sigma}\right)^{2} u=-u+\cdots
$$

where $\Delta_{\sigma}$ is the Laplace-Beltrami operator on $S^{N-1}$. Indeed, using the representation

$$
u(x)=R(r) Y(\sigma)+\cdots,
$$

with $R(r)$ obtained above, it then leads to a complicated equations on $Y(\sigma)$. In general, such an asymptotic separation procedure is expected to determine a sufficiently wide infinitedimensional asymptotic bundle of solutions with an exponential decay at infinity.

However, we do not need such a full and rather technical analysis. We must admit that we still do not know that whether a countable family of L-S critical points are radially symmetric solutions or not. If the former is true, then the above radial analysis is sufficient. In general, using our previous experience, we expect that min-max critical points are not all radially symmetric, but cannot prove that.

Finally, we note that, obviously, this is not the case in 1D. Indeed, in addition to the symmetric (even) profiles satisfying (2.4), there exist others dipole-like/anti-symmetric (odd) solutions such that

$$
u(0)=u^{\prime \prime}(0)=0
$$

We can also check existence of such solutions numerically; see Section 5.

\subsection{Spectral theory in $\mathbb{R}^{N}$}

Now, we consider the linear spectral problem for the corresponding linearised non-local equation (1.5), i.e.,

$$
\mathbf{L} \psi_{\beta} \equiv-\Delta \psi_{\beta}+(-\Delta)^{-1} \psi_{\beta}=\lambda_{\beta} \psi_{\beta} \quad \text { in } \mathbb{R}^{N}, \quad \lim _{|x| \rightarrow \infty} \psi_{\beta}(x)=0,
$$


which was analysed, however, in a bounded domain, in full detail in [9]. In problem (2.8) $\lambda_{\beta}$ represents the $\beta$ th-eigenvalue associated with the eigenfunction $\psi_{\beta}$.

In the following we show and prove several properties of the spectrum of the linear eigenvalue problem (2.8). Subsequently, we will apply them, in particular, in order to get estimations of the category; see Section 4.

As usual, we begin checking that the linearised equation (2.8) admits solutions with a proper exponential decay at infinity. Indeed, writing it down in the equivalent form

$$
\left\{\begin{array}{l}
\Delta^{2} \psi_{\beta}+\psi_{\beta}=-\lambda_{\beta} \Delta \psi_{\beta} \quad \text { in } \mathbb{R}^{N} \quad \text { and } \\
\lim _{|x| \rightarrow+\infty} \psi_{\beta}(x)=0
\end{array}\right.
$$

we see that a proper exponential decay at infinity holds (here, as before, $r=|x| \gg 1$, so we are again restricted to the radial setting of this linear spectral problem).

Moreover, due to the positivity of the operator $\mathbf{L}=\Delta^{2}+$ Id on the left-hand side of (2.9), $\lambda_{\beta}=0$ is not an eigenvalue, so that $\lambda_{\beta}>0$, for any $\beta$. Secondly, owing to Theorem VI.8 of [27] and the compactness of the inverse integral operator the spectrum might contain either infinitely many isolated real eigenvalues or a finite number of isolated eigenvalues, formed by a monotone sequence of eigenvalues

$$
0<\lambda_{1} \leq \lambda_{2} \leq \cdots \leq \lambda_{\beta} \leq \cdots
$$

In other words, for a sufficiently large $\alpha$, the resolvent of the operator $\mathbf{L}+\alpha \mathrm{I}$ is compact and, hence, the spectrum is discrete and, of course, real, by symmetry (self-adjoint). Note as well that when the operator is self-adjoint the method shown in [28] can be used to prove that there are infinitely many eigenvalues. Thus, we have the following result.

Proposition 2.1 The operator $\mathbf{L}$ admits a discrete set of eigenvalues that tend to $+\infty$ and there exists at least a solution $\psi \in W_{\mathrm{rad}}^{1,2}\left(\mathbb{R}^{N}\right)$ for problem (2.9).

\section{Remarks}

- According to our analysis above, to get exponentially decaying solutions, the real eigenvalues in (2.8) must satisfy

$$
\lambda_{\beta}>0 \text { for any } \beta \text {. }
$$

Note that problem (2.9) admits a positive first eigenvalue $\lambda_{1}$ characterised by the Raileigh quotient

$$
\lambda_{1}:=\inf _{u \in W_{\text {rad }}^{2,2}\left(\mathbb{R}^{N}\right)} \frac{\int_{\mathbb{R}^{N}}|\Delta u|^{2}+\int_{\mathbb{R}^{N}} u^{2}}{\int_{\mathbb{R}^{N}}|\nabla u|^{2}} .
$$

- Furthermore, the strict positivity of eigenvalues, for eigenfunctions with an exponential decay at infinity follows directly from the equality

$$
\int\left|\Delta \psi_{\beta}\right|^{2}+\int\left|\psi_{\beta}\right|^{2}=\lambda_{\beta} \int\left|\nabla \psi_{\beta}\right|^{2}
$$

obtained after multiplying 2.9 by $\psi_{\beta}$ and integrating in $L^{2}$. 
- Moreover, the corresponding associated family of eigenfunctions $\left\{\psi_{\beta}\right\}$ is a complete orthogonal set in $W^{2,2}$.

Also, we find the following simple observation:

Proposition 2.2 If $\lambda_{\beta}>0$ is an eigenvalue of (2.9) for any $\beta$, then

$$
\lambda_{\beta} \geq 2 \text {. }
$$

Proof Indeed, from (2.12), integrating by parts and applying the Hölder inequality yields

$$
\begin{aligned}
\left|\int \nabla \psi_{\beta} \cdot \nabla \psi_{\beta}\right| & =\left|\int \Delta \psi_{\beta} \psi_{\beta}\right| \leq\left(\int\left|\Delta \psi_{\beta}\right|^{2}\right)^{\frac{1}{2}}\left(\int \psi_{\beta}^{2}\right)^{\frac{1}{2}} \\
& \leq \frac{1}{2}\left[\int\left(\left|\Delta \psi_{\beta}\right|^{2}+\psi_{\beta}^{2}\right)\right],
\end{aligned}
$$

which proves the proposition.

Furthermore, the natural space for the eigenfunctions of problem (2.9) is $W_{\mathrm{rad}}^{2,2}\left(\mathbb{R}^{N}\right)$, i.e., the closure of $C_{0}^{\infty}$-functions with respect to the norm

$$
\|u\|_{W^{2,2}\left(\mathbb{R}^{N}\right)}^{2}=\int_{\mathbb{R}^{N}}|\Delta u|^{2}+\int_{\mathbb{R}^{N}}|\nabla u|^{2}+\int_{\mathbb{R}^{N}} u^{2},
$$

and the associated inner product

$$
\langle u, v\rangle=\int_{\mathbb{R}^{N}} \Delta u \Delta v+\int_{\mathbb{R}^{N}} \nabla u \nabla v+\int_{\mathbb{R}^{N}} u v .
$$

Indeed, the space $W_{\text {rad }}^{2,2}\left(\mathbb{R}^{N}\right)$ is a reflexive Hilbert space.

Under those assumptions we have the following variational expression of problem (2.9):

$$
\int_{\mathbb{R}^{N}} \Delta \psi_{\beta} \Delta v+\int_{\mathbb{R}^{N}} \psi_{\beta} v=\lambda_{\beta} \int_{\mathbb{R}^{N}} \nabla \psi_{\beta} \cdot \nabla v \quad \text { for any } v \in W_{\text {rad }}^{2,2}\left(\mathbb{R}^{N}\right) \text {, for any } \beta \text {. }
$$

Thus, $\psi_{\beta} \in W_{\text {rad }}^{2,2}\left(\mathbb{R}^{N}\right) \backslash\{0\}$ is an eigenfunction of the problem (2.9) associated with the eigenvalue $\lambda_{\beta}$.

\section{Mountain pass theorem and existence of at least one solution}

In this section, we apply the celebrated mountain pass theorem $(c f .[23,29]$ for details of this highly cited theorem) to ascertain the existence of a solution for problem (1.4) in $\mathbb{R}^{N}$.

Recall that, in $\mathbb{R}^{N}$, with any $N \geq 2$, we are restricted to a class of radially symmetric solutions that belong to the space $\mathbf{H}$ denoted by (1.7) and for which we know their exponential decay at infinity. For $N=1$, we can deal with both even and odd patterns. However, in general, restrictions to both symmetries or not, makes no difference in the variational analysis. Nevertheless, in order to have the compact Sobolev's embedding (1.12) in the subcritical range (1.10) our results in this section are restricted to $N \geq 2$. Note that for $N=1$ one could argue to the possibility of getting that compact with some extra conditions in the cone of positive radially decreasing functions, however, here we cannot be assured of the positivity of the solutions. 


\subsection{Mountain pass theorem to ascertain the existence of a solution for problem (1.4)}

To obtain the existence of solutions for the stationary Cahn-Hilliard equation (1.4) we apply the mountain pass theorem to equation (1.5). Hence, we look for critical points of the functional $\mathcal{F}(u)$ (1.6) which correspond to weak solutions of equation (1.5), i.e.,

$$
\int_{\mathbb{R}^{N}} \nabla u \cdot \nabla \varphi+\int_{\mathbb{R}^{N}}(-\Delta)^{-\frac{1}{2}} u \cdot(-\Delta)^{-\frac{1}{2}} \varphi-\int_{\mathbb{R}^{N}}|u|^{p-1} u \varphi=0
$$

for any $\varphi \in W_{\text {rad }}^{1,2}\left(\mathbb{R}^{N}\right) \equiv H_{\text {rad }}^{1}\left(\mathbb{R}^{N}\right)\left(\right.$ or $\left.C_{0}^{\infty}\left(\mathbb{R}^{N}\right)\right)$ a radial function. However, by elliptic regularity, those weak solutions $u$ are also strong solutions of equation (1.5); see [30].

Then we look for the existence of critical points for the functional $\mathcal{F}(u)(1.6)$. As previously proved in [1] this functional is weakly lower semicontinuous and its Fréchet derivative has the expression

$$
D_{u} \mathcal{F}(u) \varphi:=\int_{\mathbb{R}^{N}} \nabla u \cdot \nabla \varphi+\int_{\mathbb{R}^{N}}(-\Delta)^{-\frac{1}{2}} u \cdot(-\Delta)^{-\frac{1}{2}} \varphi-\int_{\mathbb{R}^{N}}|u|^{p-1} u \varphi, \quad \varphi \in H_{\mathrm{rad}}^{1}\left(\mathbb{R}^{N}\right),
$$

such that the directional derivative (Gâteaux's derivative) of the functional (1.6) is the following:

$$
\frac{\mathrm{d}}{\mathrm{d} t} \mathcal{F}(u+t \varphi)_{\mid t=0}=\left\langle D_{u} \mathcal{F}(u), \varphi\right\rangle=D_{u} \mathcal{F}(u) \varphi .
$$

Moreover, we know that the functional is $C^{1}$ and the critical points of the functional (1.6) denoted by

$$
\mathcal{C}:=\left\{u \in W_{\text {rad }}^{1,2}\left(\mathbb{R}^{N}\right): D_{u} \mathcal{F}(u) \varphi=0 \text { for any } \varphi \in W_{\text {rad }}^{1,2}\left(\mathbb{R}^{N}\right)\right\}
$$

are weak solutions in $H_{\mathrm{rad}}^{1}\left(\mathbb{R}^{N}\right)$ for equation (1.5), i.e.,

$$
D_{u} \mathcal{F}(u) \varphi=0 .
$$

Thus, $u \in \mathcal{C}$ then

$$
\mathcal{F}^{\prime}(u):=\int_{\mathbb{R}^{N}}|\nabla u|^{2}+\int_{\mathbb{R}^{N}}\left|(-\Delta)^{-\frac{1}{2}} u\right|^{2}-\int_{\mathbb{R}^{N}}|u|^{p+1}=0,
$$

where $\mathcal{F}^{\prime}$ is called the gradient of $\mathcal{F}$ at $u$. Again, by classic elliptic regularity (Schauder's theory; see [30] for further details) we will then always obtain classical solutions for such equations.

The main ingredient we are applying in getting the existence of a solution is the celebrated mountain pass theorem due to Ambrosetti-Rabinowitz [23]. Before applying the mountain pass theorem, we show a sufficient condition in order to find a critical point via the mountain pass theorem. This is the so-called Palais-Smale condition.

\subsection{Palais-Smale condition (PS)}

Let $E$ be a Banach space and $\left\{u_{n}\right\} \subset E$ a sequence such that

$$
\mathcal{F}\left(u_{n}\right) \text { is bounded and } \mathcal{F}^{\prime}\left(u_{n}\right) \rightarrow 0 \text { as } n \rightarrow \infty \text {, }
$$


then $\left\{u_{n}\right\}$ is pre-compact, i.e., $\left\{u_{n}\right\}$ has a convergent subsequence. In particular,

$$
\mathcal{F}\left(u_{n}\right) \rightarrow c \text { and } \mathcal{F}^{\prime}\left(u_{n}\right) \rightarrow 0 \Rightarrow\left\{u_{n}\right\} \text { has a convergent subsequence. }
$$

The (PS) condition is a convenient way of imposing some kind of compactness into the functional $\mathcal{F}$. Indeed, this (PS) condition implies that the set of critical points at the level value $c$

$$
\mathcal{C}_{c}=\left\{u \in E ; \mathcal{F}(u)=c, \mathcal{F}^{\prime}(u)=0\right\}
$$

is compact for any $c \in \mathbb{R}$.

Remark 3.1 Since the functional $\mathcal{F}$ is $C^{1}$ it is easily proved that if there exists a minimizing sequence $\left\{u_{n}\right\}$ for the functional $\mathcal{F}$ weakly convergent in $H_{\text {rad }}^{1}\left(\mathbb{R}^{N}\right)$ to certain $u_{0} \in H_{\text {rad }}^{1}\left(\mathbb{R}^{N}\right)$ and such that $\mathcal{F}^{\prime}\left(u_{n}\right) \rightarrow 0$ then we can be assured that $u_{0}$ is a critical point, i.e., $\mathcal{F}^{\prime}\left(u_{0}\right)=0$.

Remark 3.2 As Ambrosetti-Rabinowitz mentioned, on a heuristic level, the mountain pass theorem says that, if a pair of points in the graph of $\mathcal{F}$ are separated by a mountain range there must be a mountain pass containing a critical point between them. Also, although the statement of the theorem does not imply it, normally in the applications the origin $u=0$ is a local minimum for the functional $\mathcal{F}$. As we will see below that is our case.

Most of the critical points will be maxima or minima. However, we cannot be assured directly that those critical points are global maxima or minima, we shall need to work a bit harder to obtain that.

Thus, we first show that for our problem the trivial solution $u=0$ is a local minimum.

Lemma 3.1 The functional $\mathcal{F}(u)$ defined by (1.6) possesses a local minimum at $u=0$.

Proof Take a function $g \in H_{\mathrm{rad}}^{1}\left(\mathbb{R}^{N}\right)$ normalised in the following way:

$$
\int_{\mathbb{R}^{N}}|\nabla g|^{2}=1
$$

Then, taking a real number $t$ sufficiently close to 0 , using the expression of the functional $\mathcal{F}$ and applying the compact Sobolev embedding (1.12), (1.10) we find that

$$
\mathcal{F}(t g)=\frac{t^{2}}{2}+\frac{t^{2}}{2} \int_{\mathbb{R}^{N}}\left|(-\Delta)^{-\frac{1}{2}} g\right|^{2}-\frac{t^{p+1}}{p+1} \int_{\mathbb{R}^{N}}|g|^{p+1} \geq\left(\frac{t^{2}}{2}-\frac{t^{p+1} K}{p+1}\right)
$$

for a constant $K>0$ that depends on the Sobolev constant $S_{N}$. Then we arrive at

$$
\mathcal{F}(t g) \geq\left(\frac{t^{2}}{2}-\frac{t^{p+1} K}{p+1}\right)>0=\mathcal{F}(0),
$$

with $t$ sufficiently close to zero, i.e.,

$$
t^{p-1}<\frac{p+1}{2 K}
$$

As a first step we prove that the (PS) condition is satisfied by the functional $\mathcal{F}$ (1.6). 
Lemma 3.2 The functional $\mathcal{F}$ denoted by (1.6) satisfies the Palais-Smale condition.

Proof Let $\left\{u_{n}\right\}$ be a sequence of radial functions such that $\mathcal{F}\left(u_{n}\right) \rightarrow c$ and $\mathcal{F}^{\prime}\left(u_{n}\right) \rightarrow 0$. Then, since $\mathcal{F}^{\prime}\left(u_{n}\right) \rightarrow 0$, for any $\varepsilon>0$, there exists a subsequence of $\left\{u_{n}\right\}$ (denoted again by $\left.\left\{u_{n}\right\}\right)$ such that

$$
\left.\left|\int_{\mathbb{R}^{N}} \nabla u_{n} \cdot \nabla \varphi+\int_{\mathbb{R}^{N}}(-\Delta)^{-\frac{1}{2}} u_{n} \cdot(-\Delta)^{-\frac{1}{2}} \varphi-\int_{\mathbb{R}^{N}}\right| u_{n}\right|^{p-1} u \varphi \mid \leq \varepsilon\|\varphi\|_{W_{\mathrm{rad}}^{1,2}\left(\mathbb{R}^{N}\right)} .
$$

Indeed, $\varphi=u_{n}$ yields

$$
\left.\left|\int_{\mathbb{R}^{N}}\right| \nabla u_{n}\right|^{2}+\int_{\mathbb{R}^{N}}\left|(-\Delta)^{-\frac{1}{2}} u_{n}\right|^{2}-\int_{\mathbb{R}^{N}}\left|u_{n}\right|^{p+1} \mid \leq \varepsilon\left\|u_{n}\right\|_{W_{\mathrm{rad}}^{1,2}\left(\mathbb{R}^{N}\right)} .
$$

Furthermore, due to (3.2) we see that $\mathcal{F}\left(u_{n}\right)$ is bounded (or $\mathcal{F}\left(u_{n}\right) \rightarrow$ c), i.e.,

$$
\begin{aligned}
& \left|\mathcal{F}\left(u_{n}\right)\right| \leq c \quad \text { or } \\
& \frac{1}{2} \int_{\mathbb{R}^{N}}\left|\nabla u_{n}\right|^{2}+\frac{1}{2} \int_{\mathbb{R}^{N}}\left|(-\Delta)^{-\frac{1}{2}} u_{n}\right|^{2}-\frac{1}{p+1} \int_{\mathbb{R}^{N}}\left|u_{n}\right|^{p+1}=c+o(1),
\end{aligned}
$$

Hence, for a positive constant $\mu \in \mathbb{R}$ (to be chosen below) and using (3.3), (3.4)

$$
\mu \mathcal{F}\left(u_{n}\right)-\left\langle D_{u} \mathcal{F}\left(u_{n}\right), u_{n}\right\rangle=c+o(1)\left\|u_{n}\right\|_{W_{\mathrm{rad}}^{1,2}\left(\mathbb{R}^{N}\right)}=c+\varepsilon\left\|u_{n}\right\|_{W_{\mathrm{rad}}^{1,2}\left(\mathbb{R}^{N}\right)} .
$$

Indeed, we actually see that

$$
\begin{aligned}
& \frac{\mu-2}{2} \int_{\mathbb{R}^{N}}\left|\nabla u_{n}\right|^{2}+\frac{\mu-2}{2} \int_{\mathbb{R}^{N}}\left|(-\Delta)^{-\frac{1}{2}} u_{n}\right|^{2}+\left(1-\frac{\mu}{p+1}\right) \int_{\mathbb{R}^{N}}\left|u_{n}\right|^{p+1} \\
& =c+\varepsilon\left\|u_{n}\right\|_{W_{\mathrm{rad}}^{1,2}\left(\mathbb{R}^{N}\right)} .
\end{aligned}
$$

Subsequently, choosing $\mu$ bigger than 2 but sufficiently close to it, i.e., for $2 \delta+2<\mu<p+1$, with $\delta>0$ we arrive at the inequality

$$
\delta\left\|u_{n}\right\|_{W_{\mathrm{rad}}^{1,2}\left(\mathbb{R}^{N}\right)}^{2} \leq c+\varepsilon\left\|u_{n}\right\|_{W_{\mathrm{rad}}^{1,2}\left(\mathbb{R}^{N}\right)} .
$$

Consequently, for any appropriate $\varepsilon>0$, sufficiently small, we get the boundedness of the norms in $W_{\text {rad }}^{1,2}\left(\mathbb{R}^{N}\right)$ for the elements of the subsequence $\left\{u_{n}\right\}$. Consequently, we obtain the strong convergence of the sequence for a further the subsequence $\left\{u_{n}\right\}$ thanks to the Sobolev embedding (1.12), proving the (PS) condition.

Finally, we apply the mountain pass theorem in order to obtain the existence of a solution for equation (1.5). Thus, we state the following result.

Lemma 3.3 Let $\mathcal{F} \in C^{1}(\mathbf{H}, \mathbb{R})$ be the functional denoted by (1.6). Then:

(a) there exist $\rho, \alpha>0$ such that

$$
\mathcal{F}_{\partial B(0, \rho)} \geq \alpha,
$$

where $B(0, \rho)$ represents the ball centered at the origin and of radius $\rho>0$;

(b) also, there exists $e \in \mathbf{H} \backslash B(0, \rho)$ such that $\mathcal{F}(e) \leq 0$. 
Proof First, taking $u \in \mathbf{H} \backslash\{0\}$, we see that

$$
\mathcal{F}(u)=K\|u\|_{W_{\mathrm{rad}}^{1,2}\left(\mathbb{R}^{N}\right)}^{2}+o\left(\|u\|_{W_{\mathrm{rad}}^{1,2}\left(\mathbb{R}^{N}\right)}^{2}\right) \quad \text { as } u \rightarrow 0 .
$$

Indeed, since $u \in \mathbf{H}$ and due to the Sobolev embedding (1.12), in the range (1.10), yields

$$
\|u\|_{L^{p+1}\left(\mathbb{R}^{N}\right)}^{2} \leq K\|u\|_{W_{\text {rad }}^{1,2}\left(\mathbb{R}^{N}\right)}^{2} \quad \text { with } K \text { a positive constant, }
$$

so that

$$
\int_{\mathbb{R}^{N}}|u|^{p+1}=o\left(\|u\|_{W_{\mathrm{rad}}^{1,2}}^{2}\left(\mathbb{R}^{N}\right) \quad \text { as } u \rightarrow 0 .\right.
$$

Thus, we can be assured that there exist $\rho, \alpha>0$ such that

$$
\mathcal{F}_{\partial B(0, \rho)} \geq \alpha
$$

Additionally, assuming the compact Sobolev's embedding (1.12) and satisfying the decay condition at infinity (1.9), we find that

$$
\mathcal{F}(t u)=\frac{t^{2}}{2} \int_{\mathbb{R}^{N}}|\nabla u|^{2}+\frac{t^{2}}{2} \int_{\mathbb{R}^{N}}\left|(-\Delta)^{-\frac{1}{2}} u\right|^{2}-\frac{t^{p+1}}{p+1} \int_{\mathbb{R}^{N}}|u|^{p+1} \rightarrow-\infty
$$

as $t \rightarrow \infty$, for $u \in \mathbf{H}(1.7)$.

These results provide us with the existence of at least a solution for the non-local equation (1.5) and, hence, the existence of at least a stationary solution for the Cahn-Hilliard equation (1.4).

\section{Towards a first countable family of L-S critical points}

In order to estimate the number of critical points of a functional, we shall apply LusternikSchnirel'man's (L-S) classic theory of calculus of variations; see [1] and Chapters 8, 9, 10 of [29] for further details. Thus, the number of critical points of the functional (1.6) will also depend on the category of a functional subset (see below some details).

Basically, this topological theory for potential compact operators is a natural extension of the standard minimax principles which characterise the eigenvalues of linear compact self-adjoint operators. Applying the Calculus of Variations to the eigenvalue problem in an appropriate functional setting, one can see that the critical values of the functional involved are precisely the eigenvalues of the problem. Indeed, performing this characterisation in the unit sphere $S^{N-1}$ one sees that the eigenvalues are the critical points of the functional associated to a linear operator $L$ in the unit ball

$$
\partial \Sigma:=\{v:\|v\|=1\} .
$$

To extend these ideas to nonlinear potential operators, Lusternik-Schnirel'man introduced the concept of category getting a lower estimate of the number of critical points on the projective spaces. Indeed, this is estimated by the topological concept of the genus of a 
set introduced by Krasnosel'skii in the 1951 [31], avoiding the transition to the projective spaces obtained by identifying points of the sphere which are symmetric with respect to the centre, needed to estimate the category of Lusternik-Schnirel'man. Thus, the genus of a set provides us with a lower bound of the category.

Moreover, an estimate of the number of critical points of a functional is at the same time an estimate of the number of eigenvectors of the gradient functional (in Krasnosel'skii's terms) and, hence, of the number of solutions of the associated nonlinear equation.

In our particular case, this functional subset is the following:

$$
\begin{gathered}
\mathcal{R}_{0}=\left\{v \in \mathbb{H}: \mathbf{B}(v) \equiv \int_{\mathbb{R}^{N}}|\nabla v|^{2}+\int_{\mathbb{R}^{N}}\left|(-\Delta)^{-\frac{1}{2}} v\right|^{2}=1\right\}, \\
\text { with } \mathbb{H}=\left\{v \in W^{1,2}\left(\mathbb{R}^{N}\right): \int_{\mathbb{R}^{N}}\left|(-\Delta)^{-\frac{1}{2}} v\right|^{2}<\infty\right\},
\end{gathered}
$$

in the spirit of the eigenvalues of linear operators in the unit ball (4.1). According to the L-S approach (see [30,32], etc.), in order to obtain the critical points of a functional on the corresponding functional subset, $\mathcal{R}_{0}$, one needs to estimate the category $\rho$ of that functional subset. Thus, the category will provide us with the (minimal) number of critical points that belong to the subset $\mathcal{R}_{0}$. Namely, similar to [1, 12], we, formally, may use a standard result.

Lemma 4.1 The category of the manifold $\mathcal{R}_{0}$, denoted by $\rho\left(\mathcal{R}_{0}\right)$, is given by the number of eigenvalues (with multiplicities) of the corresponding linear eigenvalue problem satisfying

$$
\rho\left(\mathcal{R}_{0}\right)=\sharp\left\{\lambda_{\beta}>0\right\},
$$

where

$$
\mathbf{L} \psi_{\beta} \equiv-\Delta \psi_{\beta}+(-\Delta)^{-1} \psi_{\beta}=\lambda_{\beta} \psi_{\beta} \quad \text { in } \mathbb{R}^{N}, \quad \lim _{|x| \rightarrow \infty} \psi_{\beta}(x)=0 .
$$

Proof Let $\lambda_{\beta}$ be the $\beta$-eigenvalue of the linear bi-harmonic problem (4.4) such that

$$
\psi_{\beta}:=\sum_{k \geq 1} a_{k} \hat{\psi}_{k}
$$

taking into consideration the multiplicity of the $\beta$-eigenvalue, under the natural 'normalising' constraint

$$
\sum_{k \geq 1} a_{k}=1
$$

Here, (4.5) represents the associated eigenfunctions to the eigenvalue $\lambda_{\beta}$ and

$$
\left\{\hat{\psi}_{1}, \ldots, \hat{\psi}_{M_{\beta}}\right\}
$$

is a basis of the eigenspace of dimension $M_{\beta}$. Moreover, assume a critical point

$$
u=\sum_{k \geq 1} a_{k} \hat{\psi}_{k}
$$


belonging to the functional subset (4.2) and the eigenspace of dimension $M_{\beta}$, i.e., any real linear combination of orthonormal eigenfunctions $\left\{\hat{\psi}_{\beta}\right\}$.

Thus, substituting (4.6) (since we are looking for solutions of that form) into equation (1.5) and using the expression of the spectral problem (4.4) yields

$$
\sum_{k \geq 1} a_{k} \lambda_{k} \hat{\psi}_{k}-\left(\sum_{k \geq 1} a_{k} \hat{\psi}_{k}\right)^{p}=0,
$$

which provides us with an implicit condition for the coefficients $a_{k}$ corresponding to the critical point (4.6). Indeed, assuming normalised eigenfunctions $\psi_{\beta}$, i.e.,

$$
\int \psi_{\beta}^{2}=1
$$

and multiplying by $u$ in (1.5) and integrating we see that

$$
\sum_{k \geq 1} a_{k}^{2} \lambda_{k}-\int_{\mathbb{R}^{N}}\left(\sum_{k \geq 1} a_{k} \hat{\psi}_{k}\right)^{p+1}=0 .
$$

Furthermore, taking into account that $u \in \mathcal{R}_{0, \lambda}$ re-writing down (4.2)

$$
\mathbf{B}(u)=\int \mathbf{L} u u,
$$

and substituting into it any real linear combination of orthonormal eigenfunctions $\left\{\psi_{\beta}\right\}$ yields

$$
\sum_{k} a_{k}^{2} \lambda_{k}=1
$$

Therefore, $\mathcal{R}_{0}$ contains a sphere of an arbitrary bounded dimension. Hence, its category is then infinite.

One can say that the functional (1.6) restricted to the subset $\mathcal{R}_{0}$ is between at least two values, a maximum and a minimum one,

$$
c \leq \mathcal{F}(u) \leq c^{*}
$$

Therefore, having at least two positive critical points for such a functional and since the L-S characterisation provides us with a lower bound for solutions, but not exactly how many, we should not ruled out the situation in which there are infinitely many critical points.

Note that $\rho\left(\mathcal{R}_{0}\right)$ measures, at least, a lower bound of the total of number of L-S critical points. Moreover, thanks to the spectral theory shown above in Section 2 we have the sufficient spectral information as regards the eigenvalue problem (4.4) to obtain a sharp estimate of the category (4.3).

\subsection{L-S sequence of critical points}

Thus, we look for critical values $c_{\beta}$ denoted by

$$
c_{\beta}:=\inf _{A \in \mathcal{A}_{\beta}} \sup _{u \in A} \mathcal{F}(u) \quad(\beta=1,2,3, \ldots)
$$


corresponding to the critical points $\left\{u_{\beta}\right\}$ of the functional $\mathcal{F}(u)(1.6)$ on the set $\mathcal{R}_{0}$, where

$$
\mathcal{A}_{\beta}:=\left\{A: A \subset \mathcal{R}_{0} \text {, compact subsets, } A=-A \text { and } \rho(A) \geq \beta\right\},
$$

is the class of closed sets in $\mathcal{R}_{0}$ such that each member of $\mathcal{A}_{\beta}$ is of genus (or category) at least $\beta$ in $\mathcal{R}_{0}$. The fact that $\mathcal{A}=-\mathcal{A}$ comes from the definition of genus (Krasnosel'skii [33], p.358) such that, if we denote by $\mathcal{A}^{*}$ the set disposed symmetrically to the set $\mathcal{A}$,

$$
\mathcal{A}^{*}=\left\{v: v^{*}=-v \in \mathcal{A}\right\},
$$

then $\rho(\mathcal{A})=1$ when each simply connected component of the set $\mathcal{A} \cup \mathcal{A}^{*}$ contains neither of the pair of symmetric points $v$ and $-v$. Furthermore, $\rho(\mathcal{A})=\beta$ if each subset of $\mathcal{A}$ can be covered by a minimum, $\beta$ sets of genus one, and without the possibility of being covered by $\beta-1$ sets of genus one.

Note that just applying the definition of those critical points (4.8) we have the next result.

Lemma 4.2 (Monotonicity property of the genus) Let $c_{j}$ be the critical points defined by (4.8). Then

$$
c_{1} \leq c_{2} \leq \cdots \leq c_{\beta}
$$

with $\beta$ standing for the category of $\mathcal{R}_{0}$.

Proof Taking $\varepsilon>0$, due to definition of the critical values $c_{\beta+1}$, we see that a set $A_{1} \in \mathcal{A}_{\beta+1}$ exists, such that

$$
\sup _{v \in A_{1}} \mathcal{F}(u)<c_{\beta+1}+\varepsilon
$$

Hence, if $A_{1}$ contains a subset $A_{0} \in \mathcal{A}_{\beta}$ such that

$$
\sup _{u \in A_{0}} \mathcal{F}(u) \leq \sup _{u \in A_{1}} \mathcal{F}(v)<c_{\beta+1}+\varepsilon
$$

and

$$
c_{\beta}=\inf _{\mathcal{A} \in \mathcal{A}_{\beta}} \sup _{u \in \mathcal{A}} \mathcal{F}(u) \leq \sup _{u \in \mathcal{A}_{1}} \mathcal{F}(u)<c_{\beta+1}+\varepsilon,
$$

then

$$
c_{\beta} \leq c_{\beta+1},
$$

which completes the proof.

Roughly speaking, since the dimension of the sets $\mathcal{A}$ belonging to the classes of sets $\mathcal{A}_{\beta}$ increases with $\beta$ such that

$$
\mathcal{A}_{1} \supset \mathcal{A}_{2} \supset \cdots \supset \mathcal{A}_{\beta}
$$


this guarantees that the critical points delivering critical values (4.8) are ordered. Hence, to get those critical values we need to estimate the category $\rho$ of that set $\mathcal{R}_{0}$.

Additionally, for this functional we show the following particular result (see Chapter 9 of [29] for any further details), which provides us with a countable family of critical points for the functional $\mathcal{F}(1.6)$ in the spirit of the mountain pass theorem.

Theorem 4.1 Let $\mathcal{F} \in C^{1}(\mathbf{H}, \mathbb{R})$ be the functional defined by (1.6) with $p$ an odd number, $C^{1}$ and $\mathcal{F}(0)=0$, such that the conditions of the mountain pass theorem proved in Lemma 3.3 are satisfied. Then the functional $\mathcal{F}$ possesses a countable number of critical values.

Remark 4.1 Thus, we find a countable family of critical points of the functional $\mathcal{F}$ defined by (4.8) such that $c_{\beta}=\mathcal{F}\left(u_{\beta}\right)$ with $u_{\beta}$ a weak solution of problem (1.5). However, we cannot be assured how many exactly since so far we only acknowledge the existence of a solution obtained through the mountain pass arguments performed in the previous section.

$\mathrm{N}=1, \mathrm{p}=3$ : First even profile

(A)

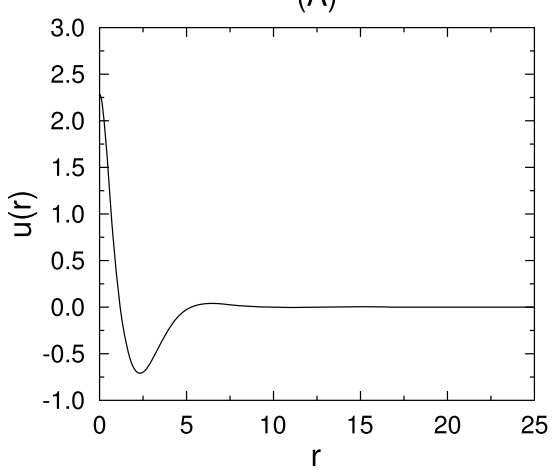

(C)

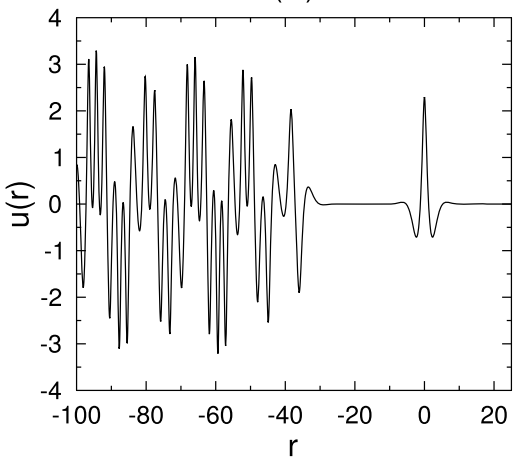

(B)

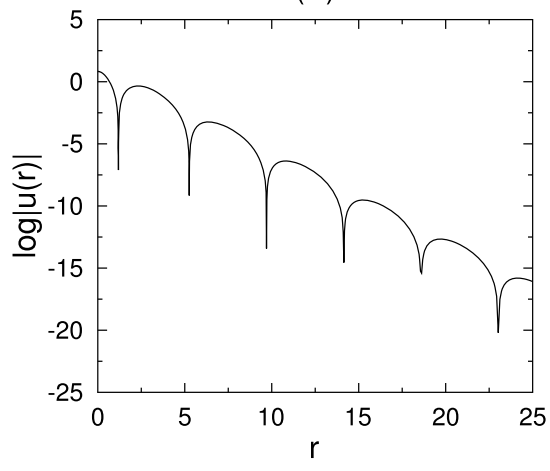

(D)

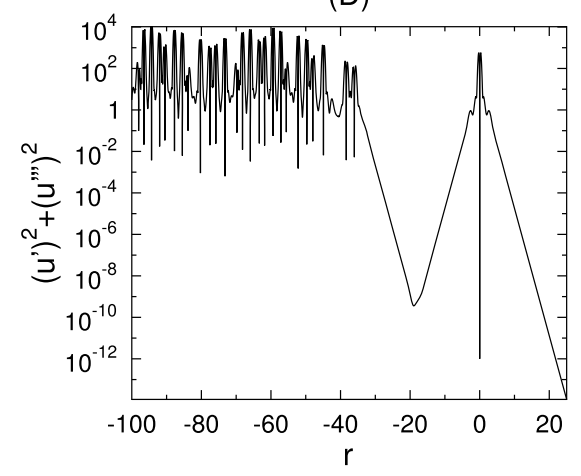

Figure 1 Numerical illustration of the first even profile for $N=1, p=3$. The profile is shown in (A), with (B) showing the oscillatory exponentially decaying tail. (C) shows the profile over the extended domain for $r<0$ emphasizing the symmetry of the solution. (D) shows the quantity $u^{\prime 2}+u^{\prime \prime \prime 2}$, which quantifies the accuracy to which the symmetry conditions are satisfied at the origin. 


\section{Numerical analysis in 1D and in the radial geometry}

Considering radial geometry as discussed in Section 2.1, (1.4) takes the form

$$
\begin{gathered}
u^{(4)}+\frac{2(N-1)}{r} u^{\prime \prime \prime}+\frac{(N-1)(N-3)}{r^{2}} u^{\prime \prime}-\frac{(N-1)(N-3)}{r^{3}} u^{\prime}+u \\
+p|u|^{p-3} u\left(u u^{\prime \prime}+(p-1)\left(u^{\prime}\right)^{2}+\frac{(N-1)}{r} u u^{\prime}\right)=0 .
\end{gathered}
$$

\section{$\mathrm{N}=1, \mathrm{p}=3$ : Selected even profiles}

(A)

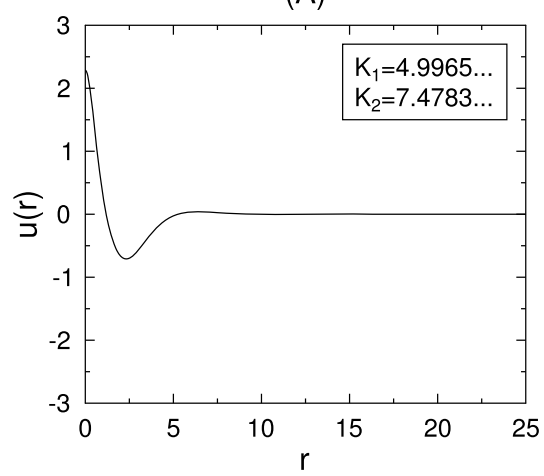

(C)

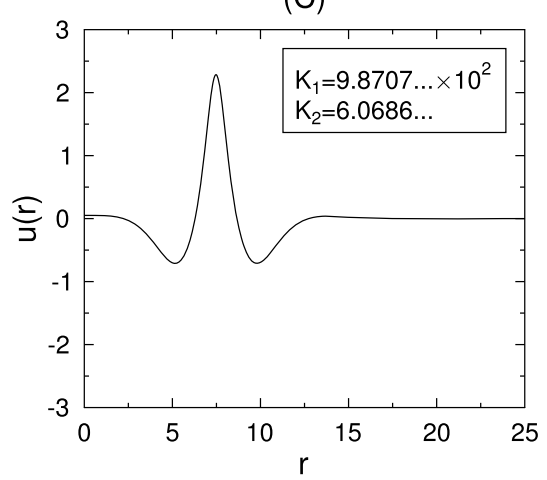

(E)

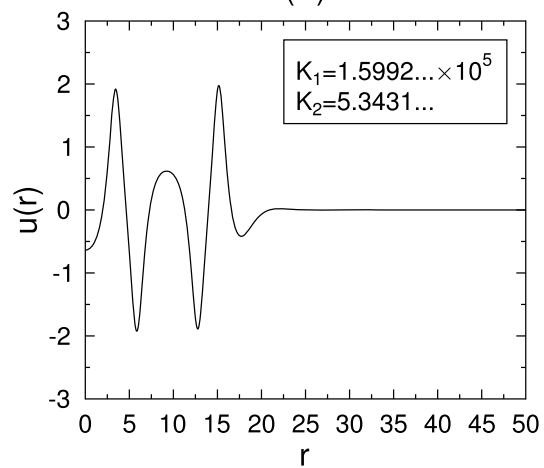

(B)

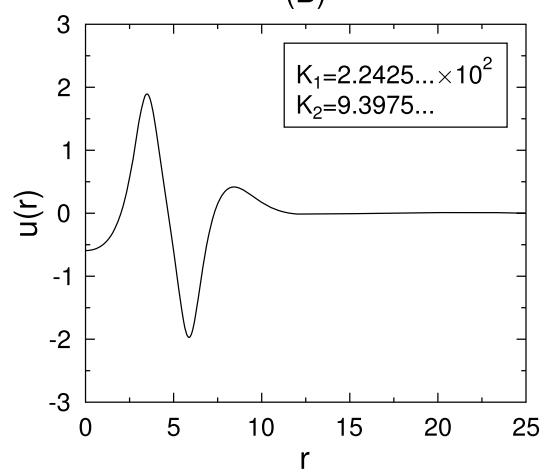

(D)

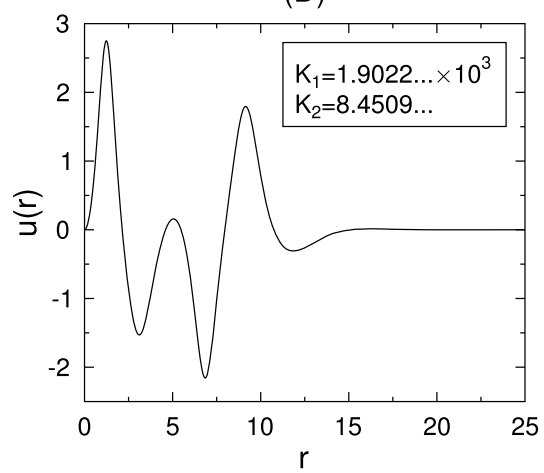

(F)

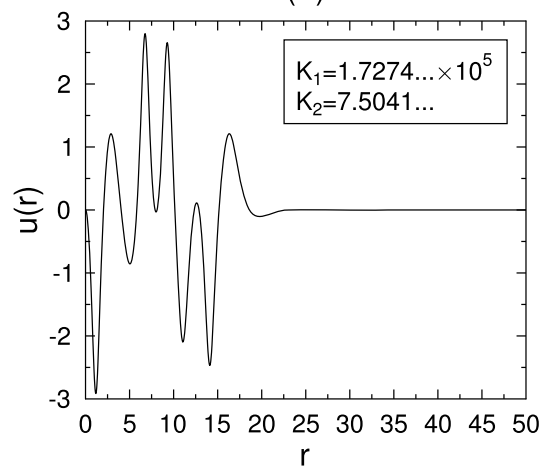

Figure 2 Illustration of even profiles for $N=1, p=3$. 

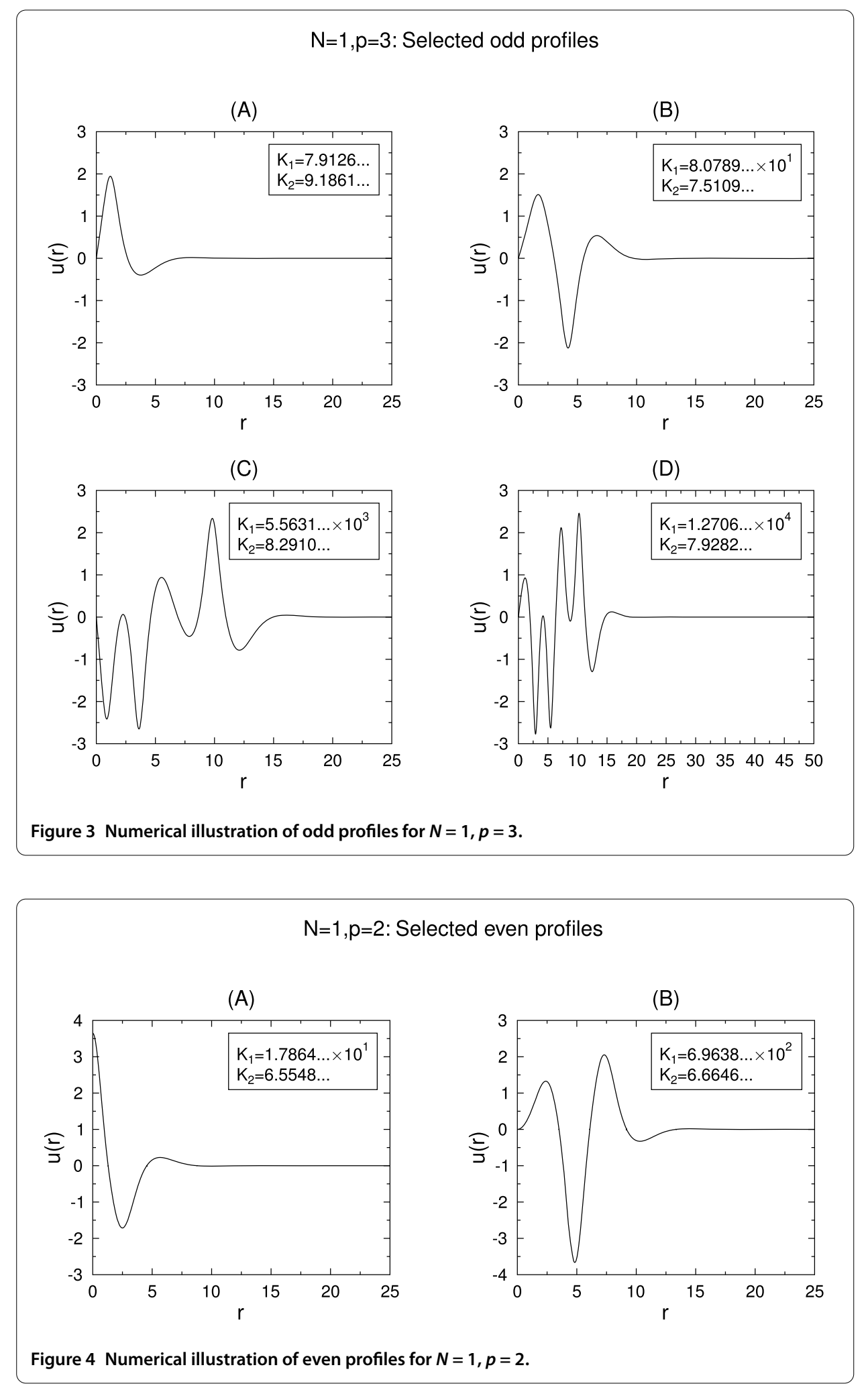
$\mathrm{N}=1, \mathrm{p}=2$ : Selected odd profiles

(A)

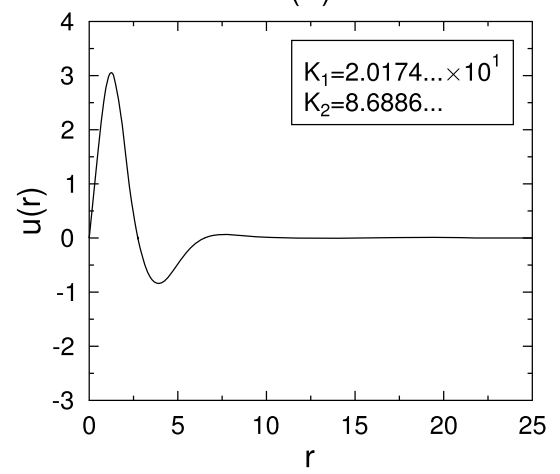

(B)

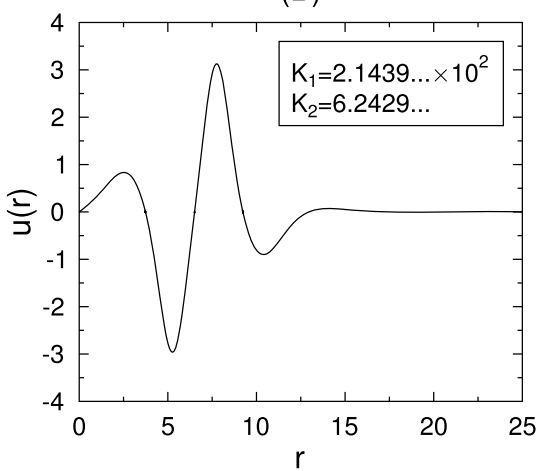

Figure 5 Numerical illustration of odd profiles for $N=1, p=2$.

\section{$\mathrm{N}=1, \mathrm{p}=3$ : Chaotic-Periodic profiles}

(A) $u(0)=1$

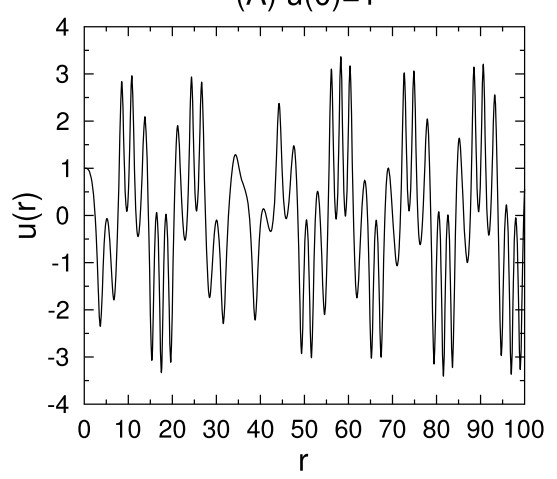

(C) $u(0)=5$

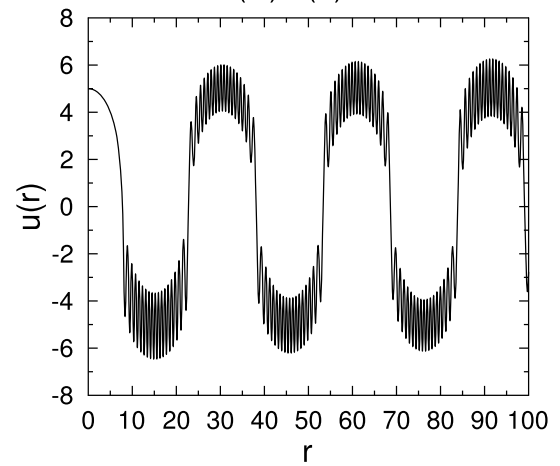

(B) $u(0)=2$

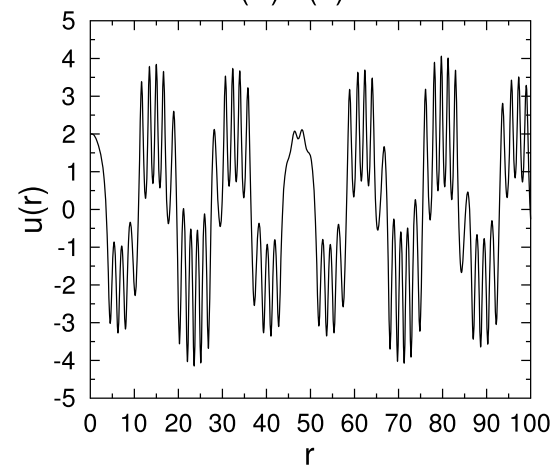

(D) $u(0)=10$

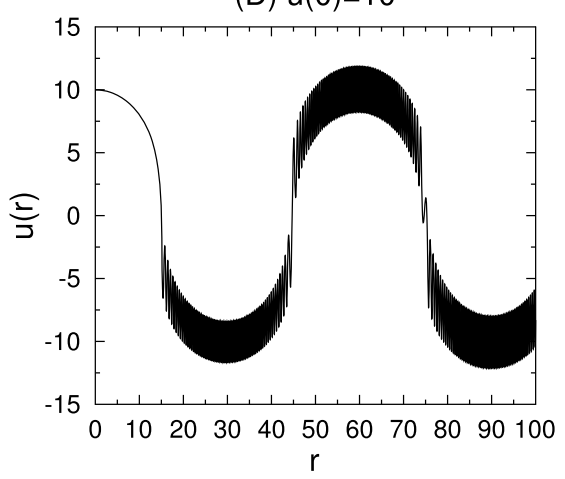

Figure 6 Numerical illustration of odd profiles for $N=1, p=2$. 


\section{$\mathrm{N}=1, \mathrm{p}=2$ : Chaotic-Periodic profiles}
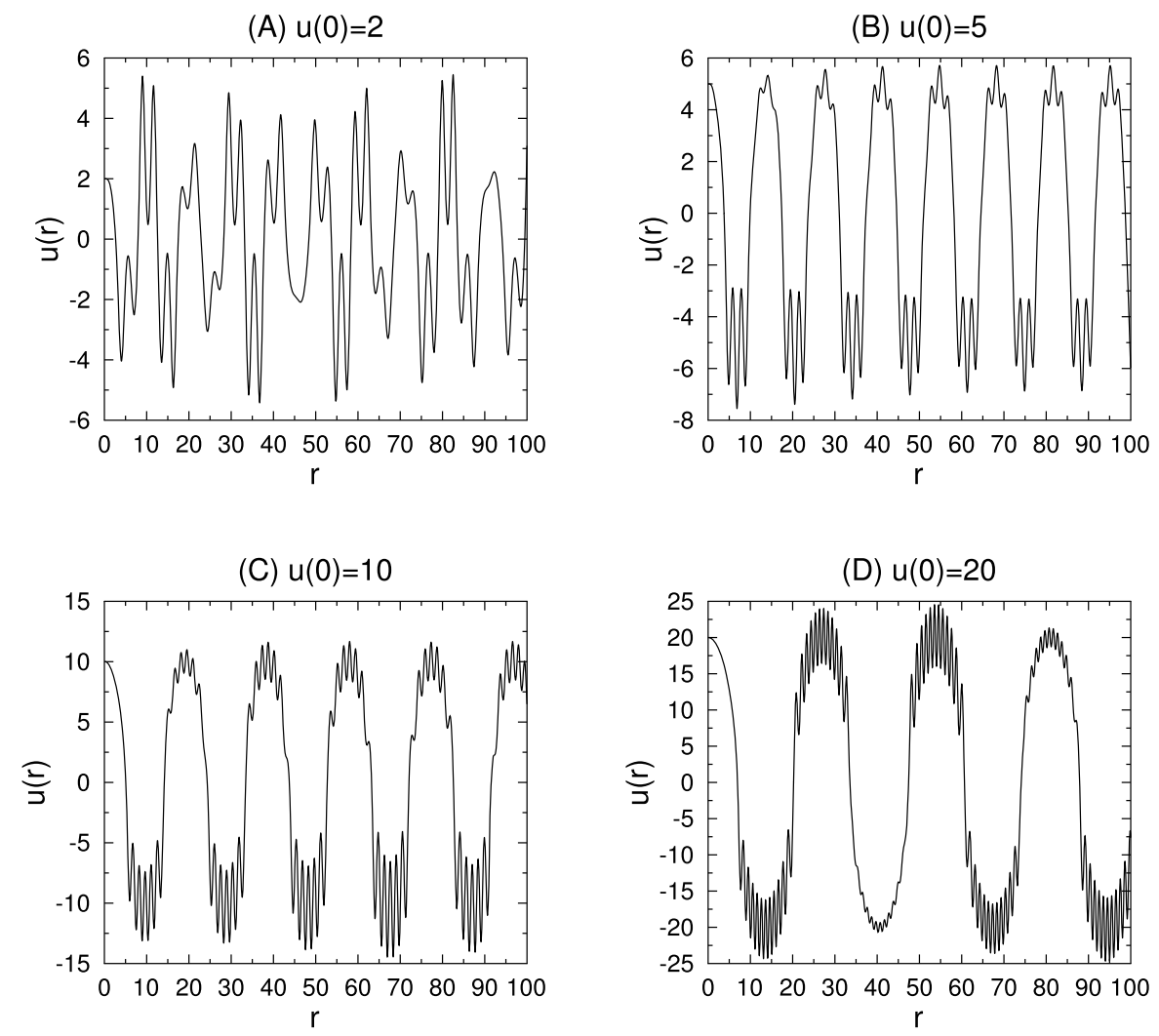

Figure 7 Numerical illustration of odd profiles for $N=1, p=2$.

This is considered together with the far-field asymptotic behaviour (2.3), written in the form

$$
\text { as } r \rightarrow \infty, \quad u \sim k_{1} r^{-\frac{(N-1)}{2}} e^{-r / \sqrt{2}} \cos \left(\frac{r-k_{2}}{\sqrt{2}}\right)
$$

where we conveniently use the constants $k_{1,2}$ in place of $C_{1,2}$, together with symmetry (2.4) or anti-symmetry (2.7) conditions at the origin.

Using the far-field behaviour (5.2), a 2D shooting problem may be formulated where the parameters $k_{1,2}$ are determined by satisfying the symmetry condition (2.4) at the origin for the even profiles and anti-symmetry condition (2.7) for the odd profiles. Matlab's ode15s solver is used with tight error tolerances ( $\left.\mathrm{RelTol}=\mathrm{AbsTol}=10^{-13}\right)$.

Figures 1-5 show illustrative even and odd profiles for $N=1$ and $p=2$ or $p=3$. The condition (5.2) is used as initial data at sufficiently large $r$ (typically 25 or 50 as given by the domain in the plots).

We complete this numerical introduction with illustration of a different class of solutions to (5.1). We may perform numerical experiments, shooting smoothly from $x=0$ with $u^{\prime}(0)=u^{\prime \prime}(0)=u^{\prime \prime \prime}(0)=0$ and varying $u(0)$. 
Shown in Figures 6 and 7 are selected profiles in one dimension $(N=1)$ in the illustrative parameter cases $p=3$ and $p=2$, respectively. For sufficiently small $u(0)$ seemingly chaotic patterns are obtained, which emerge into a more periodic structure as $u(0)$ increases.

This emergence appears sooner for $p=3$ than $p=2$ when increasing the size of $u(0)$. Such a transition behaviour is seen in similar phase solidification fourth-order equations, such as the Kuromoto-Sivashinsky and Swift-Hohenberg equations [20, 21], as a critical order parameter increases.

\section{Competing interests}

The authors declare that they have no competing interests.

\section{Authors' contributions}

All authors contributed equally to the writing of this paper. All authors read and approved the final manuscript.

\section{Author details}

${ }^{1}$ Universidad Carlos III de Madrid, Av. Universidad 30, Leganés, 28911, Spain. ${ }^{2}$ Instituto de Ciencias Matemáticas, ICMAT (CSIC-UAM-UC3M-UCM), C/Nicolás Cabrera 15, Madrid, 28049, Spain. ${ }^{3}$ Department of Mathematical Sciences, University of Bath, Bath, BA2 7AY, UK.

\section{Acknowledgements}

The authors would like to thank the anonymous referees by their valuable suggestions, helpful comments which further improved the content and presentation of the paper. Also, this work has been partially supported by the Ministry of Economy and Competitiveness of Spain under research project MTM2012-33258. The first author has also been supported by the Ramón y Cajal project RYC-2014-15284 of the Ministry of Economy and Competitiveness.

\section{Endnote}

a This is just a characteristic of a functional class: surely, we cannot use any weighted metric, where any potential approaches are lost.

Received: 16 March 2016 Accepted: 12 September 2016 Published online: 22 September 2016

\section{References}

1. Álvarez-Caudevilla, P, Galaktionov, VA: Steady states, global existence and blow-up for fourth-order semilinear parabolic equations of Cahn-Hilliard type. Adv. Nonlinear Stud. 12, 315-361 (2012)

2. Evans, JD, Galaktionov, VA, Williams, JF: Blow-up and global asymptotics of the limit unstable Cahn-Hilliard equation. SIAM J. Math. Anal. 38, 64-102 (2006)

3. Admaev, OV, Pukhnachev, W: Self-similar solutions of the equation $u_{t}+\Delta^{2} u+\Delta\left(u^{2}\right)=0$. Preprint no. 3, Comp. Centre, Krasnoyarsk (1997)

4. Novick-Cohen, A: Blow up and growth in the directional solidification of dilute binary alloys. Appl. Anal. 47, 241-257 (1992)

5. Novick-Cohen, A: The Cahn-Hilliard equation: mathematical and modeling perspectives. Adv. Math. Sci. Appl. 8 965-985 (1998)

6. Novick-Cohen, A: The Cahn-Hilliard equation. In: Handbook of Differential Equations: Evolutionary Equations, vol. IV Handb. Differ. Equat., pp. 201-228. Elsevier, Amsterdam (2008)

7. Novick-Cohen, A, Segel, LA: Nonlinear aspects of the Cahn-Hilliard equation. Physica D 10, $277-298$ (1984)

8. Galaktionov, VA, Vazquez, JL: The problem of blow-up in nonlinear parabolic equations. Discrete Contin. Dyn. Syst. 8, 399-433 (2002)

9. de Fiqueiredo, DG, Mitidieri, E: A maximum principle for an elliptic system and applications to semilinear problems. SIAM J. Math. Anal. 17, 836-849 (1986)

10. Klassen, GA, Mitidieri, E: Standing wave solutions for a system derived from the FitzHugh-Nagumo equations for nerve conduction. SIAM J. Math. Anal. 17, 74-83 (1986)

11. Galaktionov, VA, Mitidieri, E, Pohozaev, SI: Variational approach to complicated similarity solutions of higher-order nonlinear PDEs. II. Nonlinear Anal., Real World Appl. 12, 2435-2466 (2011). arXiv:1103.2643

12. Galaktionov, VA, Mitidieri, E, Pohozaev, SI: Variational approach to complicated similarity solutions of higher-order nonlinear evolution equations of parabolic, hyperbolic, and nonlinear dispersion types. In: Maz'ya, V (ed.) Sobolev Spaces in Mathematics. II: Appl. Anal. and Part. Differ. Equat. Int. Math. Ser., vol. 9. Springer, New York (2009) (an extended version in arXiv:0902.1425)

13. Strauss, WA: Existence of solitary waves in higher dimensions. Commun. Math. Phys. 55, 149-162 (1977)

14. Kalies, WD, Kwapisz, J, VanderVorst, RCAM: Homotopy classes for stable connections between Hamiltonian saddle-focus equilibria. Commun. Math. Phys. 193, 337-371 (1998)

15. Kalies, WD, Kwapisz, J, VandenBerg, JB, VanderVorst, RCAM: Homotopy classes for stable periodic and chaotic patterns in fourth-order Hamiltonian systems. Commun. Math. Phys. 214, 573-592 (2000) (Erratum: Commun. Math. Phys. 215 707-728 (2001))

16. Peletier, LA, Troy, WC: Spatial Patterns: Higher Order Models in Physics and Mechanics. Birkhäuser, Boston (2001)

17. Adams, RA, Fournier, JF: Sobolev Spaces, 2nd edn. Pure and Applied Mathematics, vol. 140. Elsevier, Amsterdam (2003)

18. Lions, PL: Symétrie et compacité dans les espaces de Sobolev. J. Funct. Anal. 49(3), 315-334 (1982) 
19. Álvarez-Caudevilla, P, Evans, JD, Galaktionov, VA: Countable families of solutions of a limit stationary semilinear fourth-order Cahn-Hilliard equation II. Non-Lusternik-Schnirel'man patterns in $\mathbb{R}$ (in preparation)

20. Paniconi, M, Elder, KR: Stationary, dynamical, and chaotic states of the two-dimensional Kuramoto-Sivashinsky equation. Phys. Rev. E 56, 2713-2721 (1977)

21. Cross, M, Greenside, H: Pattern Formation and Dynamics in Nonequilibrium Systems. Cambridge University Press, New York (2009)

22. Hoyle, R: Pattern Formation. Cambridge University Press, Cambridge (2006)

23. Ambrosetti, A, Rabinowitz, PH: Dual variational methods in critical point theory and applications. J. Funct. Anal. 14, 349-381 (1973)

24. Sato, T, Watanabe, T: Singular positive solutions for a fourth order elliptic problem in $\mathbb{R}^{N}$. Commun. Pure Appl. Anal. 10, 245-268 (2011)

25. Santra, S, Wei, J: Homoclinic solutions for fourth order traveling wave equations. SIAM J. Math. Anal. 41, 2038-2056 (2009)

26. Álvarez-Caudevilla, P: Variational approach for a class of cooperative systems. Nonlinear Anal. TMA 75, 5620-5638 (2012)

27. Brezis, H: Analyse fonctionnelle: Théorie et applications. Collection Mathématiques Appliquées pour la Maîtrise. (Collection of Applied Mathematics for the Master's Degree). Masson, Paris (1983)

28. de Figueiredo, D: Positive solutions of semilinear elliptic problems. In: Differential Equations (Sao Paulo, 1981). Lecture Notes in Math., vol. 957, pp. 34-87. Springer, Berlin (1982)

29. Rabinowitz, PH: Minimax Methods in Critical Point Theory with Applications to Differential Equations. CBMS Regional Conference Series in Mathematics, vol. 65. Am. Math. Soc., Providence (1986). Published for the Conference Board of the Mathematical Sciences, Washington, DC

30. Berger, M: Nonlinearity and Functional Analysis. Academic Press, New York (1977)

31. Krasnosel'skii, MA: Vector fields which are symmetric with respect to a subspace. Dokl. Acad. Nauk Ukrain. SSR No. 1 (1951)

32. Krasnosel'skii, MA, Zabreiko, PP: Geometrical Methods of Nonlinear Analysis. Springer, Berlin (1984)

33. Krasnosel'skii, MA: Topological Methods in the Theory of Nonlinear Integral Equations. Pergamon Press, Oxford (1964)

\section{Submit your manuscript to a SpringerOpen ${ }^{\circ}$ journal and benefit from:}

$\rightarrow$ Convenient online submission

Rigorous peer review

- Immediate publication on acceptance

Open access: articles freely available online

- High visibility within the field

- Retaining the copyright to your article 